International Journal of Database Management Systems ( IJDMS ) Vol.8, No.3, June 2016

\title{
Performance Of Data Mining Techniques To Predict In Healthcare CaSe STUdy: Chronic Kidney Failure Disease
}

\author{
Basma Boukenze ${ }^{1}$, Hajar Mousannif ${ }^{2}$ and Abdelkrim Haqiq ${ }^{3}$ \\ ${ }^{1}$ Computer, Networks, Mobility and Modeling laboratory \\ FST, Hassan 1st University, Settat, Morocco \\ 2 LISI Laboratory,FSSM Cadi Ayyad University,Marrakech 40000,Morocco \\ ${ }^{3}$ Computer, Networks, Mobility and Modeling laboratory \\ FST, Hassan 1st University, Settat, Morocco \\ e-NGN Research Group, Africa and Middle East
}

\begin{abstract}
With the promises of predictive analytics in big data, and the use of machine learning algorithms, predicting future is no longer a difficult task, especially for health sector, that has witnessed a great evolution following the development of new computer technologies that gave birth to multiple fields of research. Many efforts are done to cope with medical data explosion on one hand, and to obtain useful knowledge from it, predict diseases and anticipate the cure on the other hand. This prompted researchers to apply all the technical innovations like big data analytics, predictive analytics, machine learning and learning algorithms in order to extract useful knowledge and help in making decisions. In this paper, we will present an overview on the evolution of big data in healthcare system, and we will apply three learning algorithms on a set of medical data. The objective of this research work is to predict kidney disease by using multiple machine learning algorithms that are Support Vector Machine (SVM), Decision Tree (C4.5), and Bayesian Network (BN), and chose the most efficient one.
\end{abstract}

\section{KEYWORDS}

Predictive analytics, machine learning, big data analytics, Kidney failure disease, learning algorithm, C4.5, BN, SVM

\section{INTRODUCTION}

There is an almost universal definition shared with proponents of the ideology of big data: "Big Data sets a situation in which data sets have increased at such huge sizes that conventional technologies of information, can no longer manage them effectively, either the size or the extent and the growth of the data set" [1].

The world has become submerged by a large amount of data. Every moment is equivalent to the generation of tremendous amounts of data. All sectors and all their activities are involved due to digitization, the introduction of information technology as an effective tool, and the Internet which is becoming a very important user interface for daily interactions [2]. However, these generated data become more and more difficult to manage in terms of volume, variety and velocity [3]. This gave birth to a new domain named big data. In 2008, Gartner used for the first time the term "Big Data" in reference to the explosion of digital data and quoted it will impact the way we work [4].

"Big Data" and "analysis of big data" are inseparable. This reflects the common opinion that "Big data" does not refer to the problem of information overload, but refers also to the analytical tools used to manage the flow of data and transform the flood in a source of useful information.

DOI: $10.5121 / \mathrm{ijdms} .2016 .8301$ 
The medical field has its great contribution in this deluge of data because of some technological innovations in the field, like cloud computing which has relocated the tests of care beyond the four walls of the hospital, and has made them available anywhere and anytime [5], laparoscopic surgery and robotic surgery, which replaced classical surgery [6], and smart homes which allow patients self-care and monitoring using simple devices that deliver results on specific physiological conditions. There are also smart applications or software that can analyze the body signals using integrated sensors with the aim of monitoring [7], as well as mHealth technologies that support new methods of biological, behavioural and environmental data collection. These include sensors that monitor the phenomena with high accuracy [8].

All these innovations participated to the explosion of medical data, by multiplying data sources and electronic medical records containing diagnostic Images, lab results, and biometric information that are generated and stored [8.9.10].

Researchers have deduced that this explosion of medical data has the potential to improve clinical decisions at the point of care. Doctor will become able to extract relevant knowledge for each patient, which gives better decisions and results [11].

On most of this, the term "analyzing medical data" and "predictive analytics" in Google Trends showed an impressive growth of interest from 2011 [12], because the process of analysis in the medical sector does not stop just at the level of the ability to manage large databases, but it exceeds this to the ability to retrieve future knowledge, which is encouraged by many researchers and experts. Seen that an analysis of the big data shows itself as the only solution able to solve all the problems of the medical sector [13] by:

- Providing better Services

- Monitoring quality in hospital

- Improving treatment processes

- Detecting diseases earlier

There are many algorithms for classification and prediction that can be applied to predict diseases like breast cancer, heart disease, motor neuron, and diabetes. In this present paper, we apply a decision tree classifier (C4.5) [14], which is among the most influential data mining algorithm in the research community and among the top 10 data mining algorithms. Our aim is to predict chronic kidney disease by this learning algorithm.

The rest of this paper is organized as follows:

- Section 2 is about related work.

- Section 3 presents the context of the experiment, metrics and research hypothesis.

- Section 4 presents Experimental results

- Section 5 discusses the results

Finally, section 6 concludes the paper.

\section{RELATED WORKS}

There is a continuous study and research going on the field of medical diagnosis. A lot of work has been done on diseases like Cancer, Diabetes, Heart attack using several data mining techniques.

Runjie Shen et .al [15] build a diagnostic model of breast cancer by using data mining techniques. A feature selection method: INTERACT is applied to select relevant features for breast cancer diagnosis, and the support vector machine is used to build the classification model. The results of the experiments show that the accuracy of the diagnostic model improves a lot by using feature selection method, in the basis of nine relevant and important features for breast 
cancer diagnosis. Through the experiments, the accuracy of the diagnostic model with feature selection is improved obviously compared with the model without feature selection.

Saravana Kumar et .al [16] use the predictive analysis algorithm in Hadoop/Map Reduce environment to predict the diabetes types prevalent, complications associated with it and the type of treatment to be provided. Based on the analysis, this system provides an efficient way to cure and care the patients with better outcomes. This research mainly focused on patients in the rural area. Treatment can be offered when it is identified in advance.

Abhishek et.al [17] have used two neural network techniques: Back Propagation Algorithm (BPA), Radial Basis Function (RBF), and one non-linear classifier Support Vector Machine (SVM) and compared them according to their efficiency and accuracy. They used WEKA 3.6.5 tool for implementation to find the best technique among the above three algorithms for Kidney Stone Diagnosis. The main purpose of their thesis work was to propose the best tool for medical diagnosis, like kidney stone identification, to reduce the diagnosis time and improve the efficiency and accuracy. From the experimental results they concluded that the back propagation (BPA) significantly improved the conventional classification technique for use in medical field.

Andrew Kusiak et.al [18] have used data pre-processing, data transformations, and data mining approach to elicit knowledge about the interaction between many of measured parameters and patient survival. Two different data mining algorithms were employed for extracting knowledge in the form of decision rules. Those rules were used by a decision-making algorithm, which predicts survival of new unseen patients. Important parameters identified by data mining were interpreted for their medical significance. They have introduced a new concept in their research work, it has been applied and tested using collected data at four dialysis sites. The approach presented in their paper reduced the cost and effort of selecting patients for clinical studies. Patients can be chosen based on the prediction results and the most significant parameters discovered.

Ashfaq Ahmed K et.al, [19] have presented a work using machine learning techniques, namely Support Vector Machine [SVM] and Random Forest [RF]. These were used to study, classify and compare cancer, liver and heart disease data sets with varying kernels and kernel parameters. Results of Random Forest and Support Vector Machines were compared for different data sets such as breast cancer disease dataset, liver disease dataset and heart disease dataset. The results with different kernels were tuned with proper parameter selection. Results were better analyzed to establish better learning techniques for predictions. It is concluded that varying results were observed with SVM classification technique with different kernel functions.

Sadik Kara et.al [20] had concentrated on the diagnosis of optic nerve disease through the analysis of pattern electroretinography (PERG) signals with the help of artificial neural network (ANN). They implemented Multilayer feed forward ANN trained with a Levenberg Marquart (LM) back propagation algorithm. The end results were classified as healthy and diseased. The stated results demonstrate that the proposed method PERG could make an effective interpretation.

With respect to all related work mentioned above, our work lays in predicting chronic kidney failure disease using C4.5, SVM and NB algorithms.

\section{EXPERIMENT}

In this work, we will apply C4.5, SVM and NB learning algorithms that will make classification and prediction on a database to extract knowledge and classify patients into two categories: chronic kidney disease (ckd) and not chronic kidney disease (notckd). 


\subsection{Experiment Environment}

In this study, we use the Waikato Environment for Knowledge Analysis (Weka). It is a comprehensive suite of Java class libraries that implement many algorithms for data mining clustering, classification, regression, analysis of results. This platform provides researchers with a perfect environment to implement and evaluate their classification model comparing to TANAGRA or ORANGE [21].

\subsection{Chronic Kidney Disease Dataset}

We used the database Chronic Kidney Disease Dataset from UCI Machine Learning Repository [22]. This database contains 400 instances and 24 integer attributes, two class (chronic kidney disease (ckd), not chronic kidney disease (notckd)). Table 1 describes the attributes of the database, while Table 2 describes the distribution of classes.

Table 1. Information Attributes

\begin{tabular}{|c|c|c|c|}
\hline Attribute & Representation & $\begin{array}{l}\text { Information } \\
\text { attribute }\end{array}$ & Description \\
\hline Age & Age & Numerical & Years \\
\hline Blood pressure & $\mathrm{Bp}$ & Numerical & $\mathrm{Mm} / \mathrm{Hg}$ \\
\hline Specific gravity & $\mathrm{Sg}$ & Nominal & $1.005,1.010,1.015,1.020,1.025$ \\
\hline Albumin & $\mathrm{Al}$ & Nominal & 0.1 .2 .3 .4 .5 \\
\hline Sugar & $\mathrm{Su}$ & Nominal & 0.1 .2 .3 .4 .5 \\
\hline Red blood cells & Rbc & Nominal & Normal, abnormal \\
\hline Pus cell & $\mathrm{Pc}$ & Nominal & Normal, abnormal \\
\hline Pus cell clumps & Pcc & Nominal & Present, notpresent \\
\hline Bacteria & $\mathrm{Ba}$ & Nominal & Present, notpresent \\
\hline $\begin{array}{ll}\text { Blood } & \text { glucose } \\
\text { random } & \end{array}$ & Bgr & Numerical & $\mathrm{Mgs} / \mathrm{dl}$ \\
\hline Blood urea & $\mathrm{Bu}$ & Numerical & $\mathrm{Mgs} / \mathrm{dl}$ \\
\hline Serum creatinin & Sc & Numerical & Mgs/dl \\
\hline Sodium & Sod & Numerical & $\mathrm{mEq} / \mathrm{L}$ \\
\hline Potassium & Pot & Numerical & $\mathrm{mEq} / \mathrm{L}$ \\
\hline Haemoglobin & Hemo & Numerical & Gms \\
\hline Packed cell volume & Pcv & Numerical & \\
\hline $\begin{array}{l}\text { White blood cell } \\
\text { count }\end{array}$ & $\mathrm{Wc}$ & Numerical & Cells/cumm \\
\hline Red blood cell count & $\mathrm{Rc}$ & Numerical & Millions/cmm \\
\hline Hypertension & Htn & Nominal & Yes, no \\
\hline Diabetes mellitus & $\mathrm{Dm}$ & Nominal & Yes, no \\
\hline $\begin{array}{ll}\text { Coronary artery } \\
\text { disease }\end{array}$ & $\mathrm{Cad}$ & Nominal & Yes, no \\
\hline Appetite & Appet & Nominal & Good, poor \\
\hline Pedal edema & $\mathrm{Pe}$ & Nominal & Yes, no \\
\hline Anemia & Ane & Nominal & Yes, no \\
\hline Class & Classe & Nominal & Ckd notckd \\
\hline
\end{tabular}

Table 2.Class Distribution

\begin{tabular}{|l|l|l|}
\hline & Class & Distribution \\
\hline $\mathbf{1}$ & Ckd & $250(62.5 \%)$ \\
\hline $\mathbf{2}$ & Notckd & $150(37.5 \%)$ \\
\hline
\end{tabular}


International Journal of Database Management Systems ( IJDMS ) Vol.8, No.3, June 2016

\subsection{Metrics and Research Hypotheses}

To understand classifier's behaviour, we use the hypotheses below:

- $\quad$ True positive (TP) is the number of positive samples correctly predicted.

- $\quad$ True negative (TN) is the number of negative samples correctly predicted

- $\quad$ False negative (FN) is the number of positive samples wrongly predicted.

- $\quad$ False positive (FP) is the number of negative samples wrongly predicted as positive.

Table 3. Metric and Research Hypotheses.

\begin{tabular}{|c|c|c|c|}
\hline Metric & Description & Formula & \\
\hline Accuracy & $\begin{array}{l}\text { Number of correct predictions from all predictions } \\
\text { made. }\end{array}$ & $\frac{T P+T N}{T P+F P+T N+F N}$ & (1) \\
\hline Sensitivity & $\begin{array}{l}\text { Proportion of positives predictions that are correctly } \\
\text { identified. }\end{array}$ & $\frac{T P}{T P+F N}$ & (2) \\
\hline Specificity & $\begin{array}{l}\text { Proportion of negatives predictions that are correctly } \\
\text { identified }\end{array}$ & $\frac{T N}{F P+T N}$ & (3) \\
\hline Precision & Positive predictive values & $\frac{T P}{T P+F P}$ & (4) \\
\hline $\begin{array}{l}\text { Mean } \\
\text { Absolute } \\
\text { Error (MAE) }\end{array}$ & $\begin{array}{l}\text { Comparison between forecasts or predictions and } \\
\text { the eventual outcomes }\end{array}$ & $\frac{F P+F N}{\mathrm{TP}+\mathrm{FP}+\mathrm{TN}+\mathrm{FN}}$ & (5) \\
\hline F-measure & Combination of precision and recall. & $\frac{2 * \text { Precision } * \text { Sensitivi } 1}{\text { Precision }+ \text { Sensitivit }}$ & (6) \\
\hline
\end{tabular}

Another important metric which is the Confusion Matrix is taken into account. It is a visualization tool that is commonly used to present the accuracy of the classifiers in classification. The columns represent the predictions, and the rows represent the actual class as shown in Table.

Table 4. Confusion Matrix Description.

\begin{tabular}{llll}
\cline { 3 - 3 } & & \multicolumn{2}{c}{ Predicted } \\
\cline { 3 - 4 } & & Positive & Negative \\
\hline Actual & Positive & TP & FN \\
& Negative & FP & TN \\
\hline
\end{tabular}

\section{EXPERIMENTAL RESUltS}

In order to test our classifier and evaluate its performance, we apply the 10-fold cross validation test which is a technique that splits the original set into a training sample to train the model, and a test set to evaluate it. After applying the pre-processing and preparation methods, we try to analyze the data visually and figure out the distribution of values in terms of performance and accuracy of the model. 
Table 5. Classifiers' Performance Criteria

\begin{tabular}{|l|l|l|l|}
\hline Evaluation criteria & C4.5 & SVM & NB \\
\hline Time to build model (s) & 0.08 & 0.41 & 0.03 \\
\hline Correctly classified instances & 396 & 391 & 380 \\
\hline Incorrectly classified instance & 4 & 9 & 20 \\
\hline Accuracy (\%) & 63 & 60.25 & 57.5 \\
\hline Error & 0.37 & 0.39 & 0.42 \\
\hline
\end{tabular}

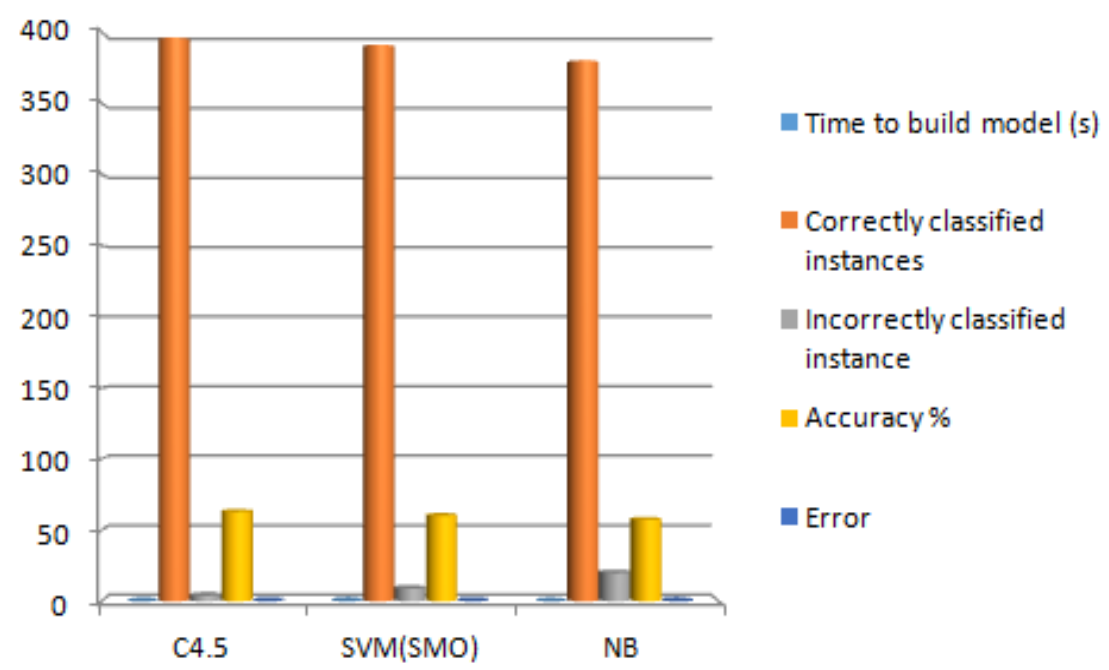

Figure 1. Comparative Graph Of Classifiers Performance

Table 6. Simulation error

\begin{tabular}{|l|c|c|c|}
\hline Evaluation criteria & C4.5 & SVM & NB \\
\hline Kappa statistic & 0.97 & 0.95 & 0.89 \\
\hline Mean absolute error & 0.02 & 0.02 & 0.04 \\
\hline Root mean squared error & 0.08 & 0.15 & 0.20 \\
\hline Relative absolute error \% & 4.79 & 4.79 & 10.21 \\
\hline Root relative squared error \% & 16.66 & 30.98 & 42.25 \\
\hline
\end{tabular}




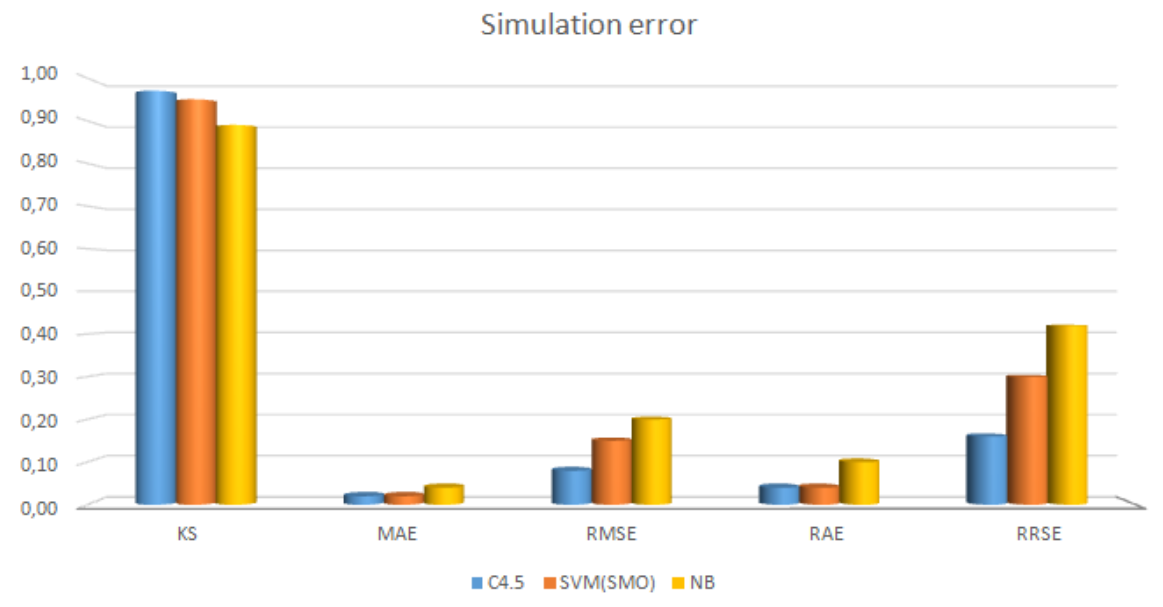

Figure 2. Comparative diagram of learning algorithms

Table 7 . Accuracy measures by class

\begin{tabular}{|c|c|c|c|c|c|c|}
\hline & \multicolumn{1}{|l|}{ TP } & FP & precision & recall & F-measure & Class \\
\hline C4.5 & 0.99 & 0.02 & 0.98 & 0.99 & 0.99 & Ckd \\
& 0.98 & 0.004 & 0.99 & 0.98 & 0.98 & Notckd \\
\hline SVM & 0.96 & 0 & 1 & 0.96 & 0.98 & Ckd \\
& 1 & 0.03 & 0.94 & 1 & 0.97 & Notckd \\
\hline NB & 0.92 & 0 & 1 & 0.92 & 0.95 & Ckd \\
& 1 & 0.08 & 0.88 & 1 & 0.93 & Notckd \\
\hline
\end{tabular}

Table 8. Confusion Matrix

\begin{tabular}{|l|c|c|c|}
\cline { 2 - 4 } \multicolumn{1}{c|}{} & Ckd & NotCkd & \multicolumn{1}{c|}{} \\
\hline \multirow{2}{*}{ C4.5 (J48) } & 249 & 1 & Ckd \\
\cline { 2 - 4 } & 3 & 147 & Notckd \\
\hline \multirow{2}{*}{ SVM(SMO) } & 241 & 9 & Ckd \\
\cline { 2 - 4 } & 0 & 150 & Notckd \\
\hline NB & 230 & 20 & Ckd \\
\cline { 2 - 4 } & 0 & 150 & Notckd \\
\hline
\end{tabular}

\section{DISCUSSION}

In this study, we applied machine learning algorithms on chronic kidney disease dataset to predict patients who have chronic kidney disease, and those who are not sick, based on the data of each attribute for each patient. Our goal was to compare different classification models and define the most efficient one. Our comparison was made on the basis of three algorithms ranked among the top 10 [23] ; SVM, NB, and C4.5, (See Fig1). The results after the implementation of classifiers on Weka show that:

$\mathrm{NB}$ is the fastest classifier because it spent the shortest time to build the classification model (0.03s) followed by C4.5. SVM is the slowest one; it took (0.41s) to build its model (see Fig2). 
Regarding accuracy, which represents the percentage of instances classified correctly, we notice a variation between $57 \%$ and $60 \%$. This has no relationship with the classifiers, but it has it with application domain and type of data. In our study, C4.5 scored a good accuracy (63\%) followed by SVM (60.25\%) and NB (57.5\%). Since Accuracy alone is not enough to define classifiers performance, we used many other criteria (see fig 2).

Regarding error rate, C4.5 marked the smallest error rate (0.37) and the largest one was scored by NB (0.42) (see Table 5). The kappa statistic value (Table 5) shows that the value of all predictors is above 0.81 . This means that our classifiers are excellent according to degree scale proposed by (Landis \& Koch) [24], except that C4.5 scored the best prediction agreement. Regarding the measurement of predictors, the values of MAE, RMSE, RAE, RRSE showed that C4.5 predictors scored the lowest values $(\mathrm{MAE}=0.02)(\mathrm{RMSE}=0.08, \mathrm{RAE}=4.79, \mathrm{RRSE}=16.66)$ followed by SVM, and NB (see fig 2).

Another important measure is F-Measures which combines two performance measures: precision and recall. If we take the case of predicted patients with the disease (ckd), C4.5 marked the best rate (0.99), and in the case of non disease (notckd) it marked the best rate also (0.98) (Table 6).

The confusion matrix (Table 8) shows us that C4.5 classified (396) instances correctly with 4 misclassified instance followed by SVM (391) and 9 instances as misclassified, then NB. C4.5 is deducted as the most efficient in terms of greatest number of instances correctly classified and the lowest error rate at the prediction. It is also the first one in accuracy and has the best f-Measures rate, with a good rate time of execution.

SVM is ranked as the second one after C4.5, but it outperforms in building time of the classification and accuracy. C4.5 has proved its performance as a powerful classifier in term of accuracy and the minimum execution time, which makes it a good classifier to be used in the medical field for classification and prediction.

\section{CONCLUSION}

As conclusion, the application of data mining techniques for predictive analysis is very important in the health field because it gives us the power to face diseases earlier and therefore save people's lives through the anticipation of cure. In this work, we used several learning algorithm C4.5, SVM and NB, to predict patients with chronic kidney failure disease (ckd), and patients who are not suffering from this disease (notckd). Simulation results showed that $\mathrm{C} 4.5$ classifier proved its performance in predicting with best results in terms of accuracy and minimum execution time.

Anticipating diseases still remains a major challenge in medical field and pushes us to increase our efforts in developing more machine learning algorithms to exploit information intelligently and extract the best knowledge from it.

\section{REFERENCES}

[1] Franck Ohlhorst, January 2013 ' Big Data Analytics: Turning Big Data into Big Money', ISBN: 9781-118-14759-7, pp 176 .

[2] Samson Oluwaseun, F., Serdar , S., and Vanduhe ,V ., ( 2014 )," Advancing big data for humanitarian needs ", Procedia Engineering ， vol . 78,N., pp 88-95

[3] Amir, G., Murtaza, H., (2015),"Beyond the hype: Big data concepts, methods, and analytics», International journal of Information Management, vol .,pp 137-144.

[4] H., Chen, H. L., Chiang, C., Storey, (2012), 'BUSINESS INTELLIGENCE AND ANALYTICS: FROM BIG DATA TO BIG IMPACT’, MIS Quarterly, Vol. 36 ,No. 4, pp. 1165-1188.

[5] Jonathan Northover, Brian McVeigh, Sharat Krishnagiri. Healthcare in the cloud: the opportunity and the challenge. MLD. Available at http://www.sunquestinfo.com/images/uploads/CMS/445/mlo_0212014_healthcare_in_the_cloud.pdf 
[6] Gabriel I. Barbas, Sherry A. Glied, (2010),' New Technology and Health Care Costs — The Case of Robot-Assisted Surgery'; the new England journal of medicine , $\mathrm{N}^{\circ}, 363$, pp 707-704 . Available at http://www.nejm.org/doi/full/10.1056/NEJMp1006602

[7] Marianthi Theoharidou, Nikos Tsalis, 'Smart Home Solutions for Healthcare: Privacy in Ubiquitous Computing Infrastructures". Available online at http://www.cis.aueb.gr/Publications/Smart\%20Home\%20-\%20Site\%20TR.pdf

[8] Steve G. Peters, James D. Buntrock,(2014),',Big Data and the Electronic Health Record',Ambulatory Care Manage , Vol. 37, No. 3, pp. 206-210

[9] R. Weil, (2014),' Big Data In Health: A New Era For Research And Patient Care Alan R. Weil', Health Affair, Vol. 33, $\mathrm{N}^{\circ}$ 7, pp 1110.

[10] Peter Groves; Basel Kayyali, ( 2013),' The 'big data' revolution in healthcare', McKinsy and Company. Center for US Health System Reform Business Technology Office. Available at http ://digitalstrategy.nl/wp-content/uploads/E2-2013.04-The-big-data-revolution-in-US-health-careAccelerating-value-and-innovation.pdf.

[11] T., Huang, L., Lan, (2015), 'Promises and Challenges of Big Data Computing in Health Sciences', Big Data Research vol. 2, pp 2-11 available at http://dx.doi.org/10.1016/j.bdr.2015.02.002

[12] Khurshid R., G., Kai, Z., John T., W., and Charles P., F., (2014), 'Harnessing Big Data for Health Care and Research Are Urologists Ready? ", Journal of European Urology, vol. N., pp 1-3

[13] Wullianallur Raghupathi,Viju Raghupathi, (2014),' Big data analytics in healthcare: promise and Potential', Health Information Science and Systems. Available at http://www.biomedcentral.com/content/pdf/2047-2501-2-3.pdf

[14] Rashedur M. Rahman, Fazle Rabbi Md. Hasan 'Using and comparing different decision tree classification techniques for mining ICDDR, B Hospital Surveillance data', Elsevier, Vol. 38, pp $11421-11436$

[15] Runjie Shen, Yuanyuan Yang and Fengfeng Shao,Intelligent Breast Cancer Prediction Model Using Data Mining Techniques, IEEE(2014), Vol.1, pp 384-387.

[16] Saravana kumar, Eswari T, Sampath P \& Lavanya S, (2015), 'Predictive Methodology for Diabetic Data Analysis in Big Data', Science direct, Vol.50, pp 203-208

[17] Abhishek, Gour Sundar Mitra Thakur, Dolly Gupta, (2012) "Proposing Efficient Neural Network Training Model for Kidney Stone Diagnosis", International Journal of Computer Science and Information Technologies, Vol. 3 (3), pp 3900-3904

[18] AndrewKusiak, Bradley Dixonb, Shital Shaha, (2005) "Predicting survival time for kidney dialysis patients: a data mining approach", Elsevier Publication, Computers in Biology and Medicine ,Vol.35, pp 311-327

[19] Ashfaq Ahmed K, Sultan Aljahdali and Syed Naimatullah Hussain, (2013) "Comparative Prediction Performance with Support Vector Machine and Random Forest Classification Techniques", International Journal of Computer Applications Vol. 69, No.11, pp 12-16

[20]Sadik Kara, Aysegul Guvenb, Ayse OztUrk Onerc, (2006) "Utilization of artificial neural networks in the diagnosis of optic nerve diseases", Elsevier Publication, Computers in Biology and Medicine, Vol. 36, pp 428-437

[21] M Hall, E Frank, G Holmes, B Pfahringer,( 2009), 'The WEKA data mining software: an update', Volume 11, Issue 1, pp 10-18

[22] "UCI Machine Learning Repository: Kidney failure Data Set [Online]. Available: https://archive.ics.uci.edu/ml/datasets/Chronic_Kidney_Disease\#

[23] http://www.datasciencecentral.com/profiles/blogs/python- resources-for-top-data-mining-algorithms

[24] Frédéric Santos, The Kappa Cohen: a tool to measure the inter-rater agreement on qualitative characters, 2015 Available at http://www.pacea.u-bordeaux1.fr/IMG/pdf/Kappa_Cohen.pdf.

\section{AUTHOR}

Basma Boukenze, PhD student at the Faculty of Science and Technology in Settat, Morroco. after obtaining a degree in computer Genie in 2009, and a master degree in engineering network and System in 2011, has continued studies and currently registered in doctoral science and technology formation at the Faculty of Science and technology Settat, Morocco, member of The Mathematical Research structure and applied computing. Laboratory Computer Networks, Mobility and modelling.

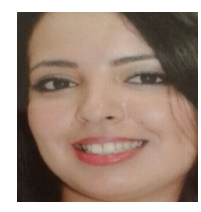

